Keywords: Mental health, Primary care, Quality of health care.

\title{
Improving the quality of mental health care in primary care settings: a view from the United Kingdom
}

\author{
Linda Gask MSc PhD FRCPsych \\ Professor of Primary Care Psychiatry \\ National Primary Care Research \\ and Development Centre \\ University of Manchester
}

UNITED KINGDOM

\begin{abstract}
Background and objectives: In the forty years since 'general practice' became a focus for research in psychiatry the UK there have been considerable developments in policy, practice and research. The aim of this paper is to review recent research and policy developments concerned with improving quality of mental health in primary care settings.

Methods: Narrative review of the literature.

Results: Disappointing results from large scales trials in the last decade have led to a move towards more exploratory studies and attempts to understand more about contextual factors. Policy initiatives such as the NICE (National Institute of Health and Clinical Excellence) guidelines have set clear standards for the delivery of care, but considerable variation in quality of care persists in primary care settings. The Medical Research Council of the UK has suggested a sequential model for future randomised trials of complex interventions.

Conclusion: Major outstanding challenges are the difficulties in recruiting GPs (General Practitioners) into research studies who are not particularly interested in mental health and linking research and policy such that the findings of such studies are effectively implemented in everyday practice.
\end{abstract}

Received 13 January 2007

Accepted 28 March 2007

It is now over forty years since 'general practice' became a focus for research psychiatry in the UK, with the pioneering work of Michael Shepherd and his colleagues at the General practice Research Unit (GPRU) at the Institute of Psychiatry ${ }^{1}$. In that time, the focus of both research and development has shifted and changed in a number of different ways: from an emphasis on detection of disorders, towards better "chronic dis- 
ease' management; from the general practitioner (GP) working alone to the partnership between the doctor, the extended primary care team and the local community; from the narrow focus of research on the behaviour of the doctor towards an exploration of the view of the patient and, in policy terms, a shift from viewing the GP as an 'independent' agent towards increasing attempts to influence the decisions that he or she makes in the assessment and management of mental health problems.

\section{Primary care mental health in the British context}

The National Health Service continues to acknowledge the central role played by the GP in mental health care. The British system, with the GP acting as gatekeeper to specialist care, maintains primary care as the key front-line provider of mental health care to the majority of people with common mental disorders, and a considerable proportion of those with more severe mental health problems ${ }^{2}$. However, the last forty years have seen vast changes in the way that mental health care is managed in both primary and specialist settings. Better training of GPs in both general communication skills and specifically in mental health care during their vocational training courses; longer appointments (not the traditional six minutes for the patient ${ }^{3}$ ) but now more commonly ten minutes under the new GP contract) improvements in prescribing practice; and a recognition of the need for training in mental health skills for the wider primary care team, including health visitors, who have a particular role in the care of postnatal depression ${ }^{4}$ and of the elderly, practice nurses $^{5}$ and community nurses ${ }^{6}$.
What however remains apparent, however, is the considerable variability in quality of care that is still provided for mental health problems in primary care. GPs continue to differ in their recognition and management of mental health problems in ways that were first described by researchers three or more decades ago $^{7,8}$. Our own research in Manchester has revealed the limited impact that the policy of 'clinical governance' -by which policy makers seek to 'assure' quality of health care and benchmark standardshas actually had on the practice of mental health care in primary care settings ${ }^{9}$. Of equal concern are the epidemiological surveys that reveal the extent of unmet need for psychiatric care in the population, and how this is not necessarily being addressed by primary care ${ }^{10}$.

\section{Approaches to quality improvement}

Campbell and his colleagues have usefully defined two principal dimensions of quality of care for individual patients; access and effectiveness ${ }^{11}$. In essence, do users of services get the care they need, and is the care effective when they get it? Within effectiveness, they define two key components-effectiveness of clinical care and effectiveness of inter-personal care. In the UK a number of randomised controlled trials of specific interventions designed to improve the effectiveness of both clinical and interpersonal care for common mental disorders (particularly depression) in terms of both clinical outcome and patient satisfaction, have been completed in the last decade, with some rather disappointing results ${ }^{12}$. Thompson and his colleagues, in the Hampshire Depression Study ${ }^{13}$, showed 
no impact from an educational intervention focussed on dissemination of a clinical treatment protocol for depression and Croudace and colleagues ${ }^{14}$ failed to demonstrate any impact of local adaptation and dissemination of ICD primary care guidelines on either rates of recognition or outcome. Our own study, which placed more emphasis on acquisition of interpersonal skills through role-play and videofeedback was also spectacularly unsuccessful ${ }^{15}$ and led us to conclude that education was in itself probably necessary but not sufficient on its own to achieve quality improvement.

In terms of the useful typology of models of quality improvement in primary care mental health reviewed by Bower and Gilbody ${ }^{16}$ (see also Table I), interventions which focus on education of the primary care team alone (as described above) would seem, at least in the UK setting, to be relatively ineffective, although the same may not be true of countries where the skills and knowledge of primary care workers are less well developed. The other major problem with such studies has been that they tend to recruit doctors who are already interested in the topic, and thus are less likely to benefit from the intervention offered. Educational interventions which are targeted at medical students and vocational trainees in primary care might be more successful in achieving change. These do seem to be key periods when (often negative) attitudes to mental health problems develop ${ }^{17}$.

Table I

Models of mental health care in primary care ${ }^{16}$.

Training primary care staff

GPs and other members of primary care team

Recognition

Pharmacological and psychological management

\section{Consultation-liaison}

Focus on improving skills of GPs

Regular specialist contact to support and feedback

Referral only after discussion

Management by primary care

\section{Collaborative care}

Training

Consultation

Case management

Direct patient contact

Education, monitoring, psychological treatment

\section{Replacement}

GP has overall clinical responsibility

Referral passes responsibility for mental health care to specialist in primary care

Specialist treatment as psychological therapy

A number of other studies have also considered replacement-referral interventions, where care, particularly for psychological therapy, involves referral onto another pro- fessional. Notable among these have been studies of counselling, cognitive-behaviour therapy and problem-solving as provided by a range of specialist professionals in the prima- 
ry care setting. King and his colleagues ${ }^{18}$ showed that psychological therapy was more effective than routine care, at least in the short-term, but there was no significant difference between counselling and CBT (which surprised many but fits with other outcome studies of CBT in primary care). Ridsdale and her colleagues ${ }^{19}$ also showed equivalence in effect between counselling and CBT for chronic fatigue in primary care. However, Kendrick et al. ${ }^{20}$ showed no impact on clinical outcome of problem-solving therapy as pro- vided by community mental health nurses for people with common mental disorders.

Recent research has thus necessarily changed tack, away from large-scale intervention studies and taken a more exploratory, step-wise approach to the development and testing of quality improvement strategies, partly at least influenced by the Medical Research Council guidance on complex interventions ${ }^{21}$ which delineates a phased approach to the development and testing of such interventions (see Table II).

Table II

Sequential phases of developing randomized controlled trials of complex interventions ${ }^{20}$.

Preclinical Explore relevant theory to ensure best choice of intervention and to predict Theory major confounders and strategic design issues.
Phase I Identify the components of the intervention and the underlying mechanisms Modelling by which they will influence the outcomes to provide evidence that you can predict how they relate to and interact with each other.

Phase II Describe the constant and variable components of a replicable intervention Exploratory trial and a feasible protocol for comparing the intervention with an appropriate alternative.

Phase III Compare a fully defined intervention with an appropriate alternative using Definitive randoa protocol that is theoretically defensible, reproducible and adequately mised controlled controlled in a study with appropriate statistical power. trial \begin{tabular}{lll}
\hline Phase IV & $\begin{array}{l}\text { Determine whether others can replicate your intervention and results in Long term imple- } \\
\text { uncontrolled settings over the long-term. }\end{array}$ \\
mentation
\end{tabular}

This approach emphasises the importance of both qualitative and theoretical work in the early stages of a programme as well as multi-method approaches to examination of outcomes in the exploratory trial phase. Our recently published study of an educational intervention for medically unexplained symptoms ${ }^{22}$ has, for example, adopted this framework as has the Enhanced Care for Depression (the ECD study ${ }^{23}$ ). In this Medical Research Council trial platform the team based in York and Manchester have carried out one of the first UK studies of collaborative care for depression. The col- laborative care model was developed by Katon, Von Korff and their colleagues ${ }^{24}$ in Seattle and is related theoretically to the development of the chronic care model by Ed Wagner and members of the same team (see www.improvingchroniccare.org). It is a truly multi-faceted complex intervention in that it includes elements of both training and consultation-liaison but also includes the addition of new quasi-specialist staff (so called case managers) who work with patients and liaise with primary care clinicians and specialists in order to improve quality of care. This model may also involve screen- 
ing, education of patients, changes in practice routines, and developments in information technology. Recent systematic reviews suggest it is a promising approach ${ }^{25}$ but it is yet to be fully tested outside the US setting. However the most promising study of collaborative care for depression was carried out in Latin America, by a British led team of researchers ${ }^{26}$.

A number of other exploratory studies of interventions for both anxiety and depression in primary care funded under the MRC brain sciences initiative have been funded and are expected to come to publication shortly.

\section{Understanding the context of care}

At alternative strand of research has however continued to inform the development and testing of interventions by helping us to understand more about the nature and outcomes of mental illness in the primary care setting. In this vein, the task carried out by the Office of National Statistics (ONS ${ }^{27}$ ) has been invaluable. However smaller scale epidemiological studies have provided information particularly about the morbidity experienced by black and ethnic minority groups living in the $\mathrm{UK}^{28,29}$.

A new strand of qualitative work in primary mental health care over the last decade has particularly focused on both patients' experience of mental disorder and helpseeking behaviour and of their experiences of mental health care as provided by their primary care providers. This has included studies on depression ${ }^{30,31}$, major mental illness $^{32}$ and the experiences of such diverse groups as Afro-caribbean women in Manchester $^{33}$ and caucasian Scottish women in
Edinburgh $^{34}$, both with post-natal depression. Several authors have also explored, some in nested qualitative studies carried out in the setting of randomised controlled trials, the barriers and potential levers for wider dissemination of interventions, in particular the views of general practitioners towards intervening with mental health problems. For example in the context of an exploratory trial of a collaborative intervention for depression in the elderly, ChewGraham and her colleagues ${ }^{35}$ have revealed major barriers to the recognition and referral of elderly patients with depression into the study, and in our own study of an educational intervention for depression in primary care, it became clear that the attitudes of GPs themselves were a major barrier against putting what they had learned on the course into everyday practice ${ }^{17}$.

\section{Research, policy and practice}

Mental health policy in primary care during the 1990s was intrinsically linked to the idea of training and education as the means by which the quality of care, particularly for depression, could be improved ${ }^{36}$. The English National Service Framework for mental health $^{37}$ was disappointing ${ }^{38}$ in its lack of specific targets for primary care in comparison both with the clear obligations placed upon secondary care mental health providers to put specific services such as Assertive Outreach services in place, and with later strategic frameworks such as those for cardiology and diabetes which were very prescriptive about what primary care should be addressing. However the NHS plan ${ }^{39}$ did include an obligation that Primary Care Trusts, the local organisations that are responsible for commissioning care in Eng- 
land and Wales, should employ and train one thousand new 'graduate mental health workers' to assist in improving access to psychological treatment in primary care settings.

The graduate worker initiative has only been partially successful ${ }^{40}$. Around seven hundred new workers are now in post, but in many places they have not been utilised to their full capacity, and integration with primary care is patchy. In some parts of England, such as the North West, where the training programme was heavily influenced by the work of Von Korff and colleagues in Seattle, the opportunity was grasped to train these new workers in the skills of case management. They are indeed well placed to play a central role in the implementation of collaborative models of care. In other places they have either remained very much in an 'assistant psychologist' role or, such as in parts of the South East and London, have not been successfully deployed at all. However the drive to improve access to psychological therapies, which addresses the second tenet of the definition of quality of care, continues with the political promise by New Labour in its last election manifesto to increase availability of psychological therapies. Although exactly how much of this will be provided in primary care or in new specialist centres remains unclear. The policy has now also become inextricably linked with the need to reduce the number of people in the UK who are receiving incapacity benefit and have a diagnosis of depression ${ }^{41}$.

Successive publications from $\mathrm{NICE}^{42}$ during the last five years (National Institute for Health and Clinical Excellence) have been influential in setting clear standards for how primary care should specifically treat common mental health problems (with guidance on depression and anxiety and the provision of computerised cognitive behav- iour therapy).This has been supported by the introduction in the new GP Quality and Outcomes Framework ${ }^{43}$, which contributes to the formula by which GPs in the UK are paid, of mental health indicators. These related initially only to improving quality of care for people with severe mental illness (particularly their physical care) and monitoring of lithium, but this year for the first time, specific indicators relating to care of depression and dementia have also been included. Of particular interest is payment for assessing the severity of new cases of depression with a recognised tool such as the Personal Health Questionnaire (PHQ9) ${ }^{44}$ and the Hospital Anxiety and Depression Scale (HADS) which may assist in ensuring that antidepressant medication is better targeted on those with moderate and severe degrees of depression ${ }^{45}$, given the evidence that GPs are not particularly good at assessments of severity ${ }^{46}$.

\section{Conclusion}

The nature of research in primary care mental health in the UK has moved away from carrying out large-scale intervention studies without first spending time developing and testing the intervention in a stepwise manner. Whether this will pay off in the longer term remains to be seen, but closer working between research centres through the Mental Health Research Network has paved the way for more efficient running of larger scale trials in better established and resourced primary care research networks. The major problem remains however of not only recruiting into such studies the GP who is not particularly interested in mental health, but also in linking research and policy such that the findings of such studies are 
effectively implemented in everyday practice. We also need to try and ensure that research into quality improvement interventions does not follow rather than precede their implementation in national policy as has so often been the case in the past, with some mixed results ${ }^{47}$.

\section{References}

1. Wilkinson G. The General Practice Research Unit at the Institute of Psychiatry. Psychol Med 1989; 19: 787-790.

2. Kendrick T, Burns T, Garland C, Greenwood N, Smith P. Are specialist mental health services being targeted on the most needy patients? The effects of setting up special services in general practice. Br J Gen Pract 2000; 50: 121-126.

3. Balint E and Norell J. Six minutes for the patient: interaction in general practice consultations. London: Tavistock publications; 1983.

4. Appleby L, Hirst E, Marshall S, Keeling F, Brind J, Butterworth $\mathrm{T}$ et al. The treatment of postnatal depression by health visitors: impact of brief training on skills and clinical practice. J Affect Disord 2003; 77: 261-266.

5. Mead N, Bower P, Gask L. Emotional problems in primary care: what is the potential for increasing the role of nurses? J Adv Nurs 1997; 26: 879-890.

6. Mayall E, Oathamshaw S, Lovell K, Pusey H. Development and piloting of a multidisciplinary training course for detecting and managing depression in the older person. J Psychiatr Ment Health Nurs 2004; 11: 165-171.

7. Shepherd M, Cooper B, Brown AC et al. Psychiatric Illness in General Practice. Second Edition Oxford: Oxford University Press; 1966.

8. Marks JN, Goldberg DP, Hillier VF. Determinants of the ability of general practitioners to detect psychiatric illness. Psychol Med 1979; 9: 337-353.

9. Gask L, Rogers A, Campbell-Sheaff R. Beyond the limits of clinical governance? The case of mental health in English primary care. (submitted for publication).

10. Bebbington PE, Brugha TS, Meltzer H, Jenkins R, Ceresa C, Farrell $\mathrm{M}$ et al. Neurotic disorders and the receipt of psychiatric treatment. Psychol Med 2000; 30: 1369-1376.

11. Campbell S, Roland MO, Buetow SA. Defining quality of care. Soc Sci Med 2000; 51: 1611-1625.
12. Gilbody S, Whitty P, Grimshaw J, Thomas R. Educational and organizational interventions to improve the management of depression in primary care: a systematic review. JAMA 2003; 289: 3145-3151.

13. Thompson C, Kinmonth AL, Stevens L, Peveler RC, Stevens A, Ostler KJ et al. Effects of a clinical practice guideline and practice-based education on detection and outcome of depression in primary care: Hampshire depression project randomized controlled trial. Lancet 2000; 355: 185-191.

14. Croudace T, Evans J, Harrison G, Sharp DJ, Wilkinson E, McCann G et al. Impact of the ICD-10 Primary Health Care (PHC) diagnostic and management guidelines for mental disorders on detection and outcome in primary care. Cluster randomised controlled trial. Br J Psychiatry 2003; 182: 20-30.

15. Gask L, Dowrick C, Dixon C, Sutton C, Perry R, Torgerson $\mathrm{D}$ et al. A pragmatic cluster randomised controlled trial of an educational intervention for GPs in the assessment and management of depression. Psychol Med 2004; 34: 63-72.

16. Bower P and Gilbody S. Managing common mental health disorders in primary care: conceptual models and evidence base. Br Med J 2005; 330: 839-842.

17. Gask L, Dixon C, May C, Dowrick C. Qualitative study of an educational intervention for general practitioners in the assessment and management of depression. Br J Gen Pract 2005; 55: 854-859.

18. Ward E, King M, Lloyd M, Bower P, Sibbald B, Farrelly $\mathrm{S}$ et al. Randomised controlled trial of non-directive counselling, cognitive-behaviour therapy, and usual general practitioner care for patients with depression. I: clinical effectiveness. Br Med J 2000; 321: 1383-1388.

19. Ridsdale L, Godfrey E, Chalder T, Seed P, King M, Wallace P. Chronic fatigue in general practice: is counselling as good as cognitive behaviour therapy? A UK randomised trial. Br J Gen Pract 2001; 51: 19-24.

20. Kendrick T, Simons L, Mynors-Wallis L, Gray A, Lathlean J, Pickering R et al. Cost-effectiveness of referral for generic care or problem-solving treatment from community mental health nurses, compared with usual general practitioner care for common mental disorders: Randomised controlled trial. Br J Psychiatry 2006; 189: 50-59.

21. Campbell M, Fitzpatrick R, Haines A. A framework for design and evaluation of complex interventions to improve health. Br Med J 2000; 321: 694-696.

22. Morriss R, Dowrick C, Salmon P, Peters S, Rogers A, Dunn $\mathrm{G}$ et al. Turning theory into practice: rationale, feasibility and external validity of an exploratory randomized controlled trial of training family practitioners in reattribution to manage patients with medically unexplained symptoms (the MUST). Gen Hosp Psychiatry 2006; 28: 343-51. 
23. Richards DA, Lankshear AJ, Fletcher J, Rogers A, Barkham M, Bower P et al. Developing a U.K. protocol for collaborative care: a qualitative study Gen Hosp Psychiatry 2006; 28: 296-305.

24. Katon W, Von Korff M, Lin E et al. Rethinking practitioner roles in chronic illness: the specialist, primary care physician and the practice nurse. Gen Hosp Psychiatry 2001; 23: 138-144.

25. Gilbody S, Bower P, Fletcher J, Richards D, Sutton AJ. Collaborative care for depression: a cumulative metaanalysis and review of longer-term outcomes. Arch Intern Med 2006; 166: 2314-2321.

26. Araya R, Rojas G, Fritsch R, Gaete J, Rojas M, Simon $\mathrm{G}$ et al. Treating depression in primary care in lowincome women in Santiago, Chile: a randomised controlled trial. Lancet 2003; 361(9362): 995-1000.

27. Singleton N, Meltzer H, Jenkins R. Building a picture of psychiatric morbidity in a nation: a decade of epidemiological surveys in Great Britain. Int Rev Psychiatry 2003; 15: 19-28.

28. Weich S, Nazroo J, Sproston K, McManus S, Blanchard M, Erens B et al. Common mental disorders and ethnicity in England: the EMPIRIC study. Psychol Med 2004; 34: 1543-1551.

29. Husain MI, Waheed W, Husain N. Self-harm in British South Asian Women: Psychosocial correlates and Strategies for Prevention. Ann Gen Psychiatry 2006; 22(5): 7.

30. Gask L, Rogers A, Oliver D, May C, Roland M. Qualitative study of patients' views of the quality of care for depression in general practice. Br J Gen Pract 2003; 53 : 278-283.

31. Lawrence V, Banerjee S, Bhugra D, Sangha K, Turner S, Murray J. Coping with depression in later life: a qualitative study of help-seeking in three ethnic groups. Psychol Med 2006; 36: 1375-1383.

32. Lester H, Tritter JQ, Sorohan H. Patients' and health professionals' views on primary care for people with serious mental illness: focus group study. Br Med J 2005; 330: 1122.

33. Edge D, Baker D, Rogers A. Perinatal depression among black Caribbean women. Health Soc Care Community 2004; 12: 430-438.

34. Maxwell M. Women's and doctors' accounts of their experiences of depression in primary care: the influence of social and moral reasoning on patients' and doctors' decisions. Chronic Illness 2005; 1: 61-71.

35. Burroughs H, Lovell K, Morley M, Baldwin R, Burns A, Chew-Graham C. 'Justifiable depression': how primary care professionals and patients view late-life depression? A qualitative study. Fam Pract 2005; 23: 369-377.

36. Paykel ES, Tylee A, Wright A, Priest RG, Rix S, Hart D. The Defeat Depression Campaign: psychiatry in the public arena. Am J Psychiatry 1997; 154 (Suppl 6): S59-S65.

37. Department of Health. A National Service Framework for Mental Health. London: Department of Health; 1999.

38. Rogers A, Campbell S, Gask L et al. Some national frameworks are more equal than others: Implementing clinical governance for mental health in primary care groups and trusts. Journal of Mental Health 2002; 2: 199-212.

39. Department of Health. The NHS Plan: a plan for investment, a plan for reform. London: HMSO; 2000.

40. Harkness E Bower P Gask L. Job satisfaction and retention of Graduate Primary Care Mental Health Workers: a newly developed role in England. J Health Serv Res Policy 2007; (in press).

41. Layard R. The case for psychological treatment centres. Br Med J 2006; 332: 1030-1032.

42. NICE guidelines. Available at: http://www.nice.org.uk

43. National Health Service [homepage on the Internet]. [Updated 2005 September 01; cited 2007 february 08] The Information Centre. Quality and Outcomes Framework Information. [about 3 screens]. Available from: http://www. ic.nhs.uk/services/qof

44. Kroenke K, Spitzer RL, Williams JB. The PHQ-9: validity of a brief depression severity measure. J Gen Intern Med 2001; 16: 606-613.

45. Kendrick T. Ever been HAD? Br J Gen Pract 2006; 56: 796-797.

46. Kendrick T, King F, Albertella L, Smith PW. GP treatment decisions for patients with depression: an observational study. Br J Gen Pract 2005; 55(513): 280-286.

47. Gravelle H, Dusheiko M, Sheaff R, Sargent P, Boaden $\mathrm{R}$, Pickard $\mathrm{S}$ et al. Impact of case management (Evercare) on frail elderly patients: controlled before and after analysis of quantitative outcome data Br Med J 2007 Jan 6; 334(7583): 31. Epub 2006 Nov 15.

Address for correspondence:

Division of Primary Care

University of Manchester

Rusholme Academic Unit

Walmer Street

Manchester

M14 5NP

Linda.Gask@manchester.ac.uk 